\title{
Localized synovial hypertrophy in the anteromedial compartment of the osteoarthritic knee
}

\author{
Saccomanni Bernardino
}

Published online: 6 July 2010

(C) Humana Press 2010

\section{To the Editor:}

In the article by Ikeuchi et al. [1] entitled 'Localized Synovial Hypertrophy in the Anteromedial Compartment of the Osteoarthritic Knee', we recognized similarities in their Fig. 1A and B, arthroscopic views of localized synovial hypertrophy in the anteromedial compartment with accompanying 'degenerated cartilage of the medial femoral condyle', to content in article published nearly 13 years before, entitled 'Meniscal Impingement Syndrome' by Mc Guire et al. [2]. Unlike the patients in the study of Chow et al. [3] where 20 of 21 patients (95\%) had a history of injury before the onset of symptoms, in the patient cohort of Ikeuchi et al., only 5 of 19 (26\%) had injury-related symptoms. Because there was a point of tenderness localized at the anteromedial joint space and the McMurray test results were positive in all patients, meniscal or cartilage injury (or both) was expected in all of their patients. Takahashi, the surgeon in all cases in the study of Ikeuchi et al. [1] found no unstable fragments of meniscus or cartilage intraoperatively in this exclusion criteria-controlled patient cohort. No meniscectomy or chondroplasty

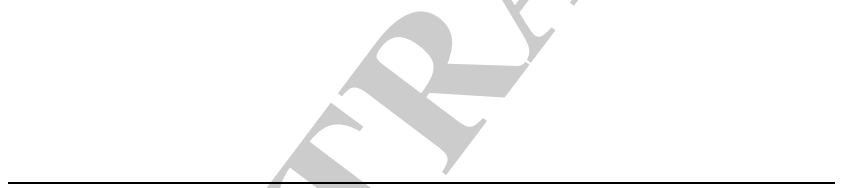

S. Bernardino $(\square)$

Orthopaedic and Traumatologic Surgery, Gabriele D' Annunzio University, Chieti (Italy), Via dei Vestini, 66013 Chieti Scalo, Italy

e-mail: bernasacco@yahoo.it

\section{S. Bernardino}

Struttura complessa di Ortopedia e Traumatologia, Azienda Sanitaria Locale Bari, Stabilimento ospedaliero di Altamura (Bari), Viale Regina Margherita n. 74, Altamura (Bari), Italy was performed in any of these patients, and the only procedures noted were partial synovectomy and joint lavage.

Unfortunately, no range-of-motion assessments were presented in their study, and in the absence of these findings, it is impossible to know whether any of these patients in this cohort, particularly those without any associated injury history, presented with meniscal impingement syndrome before incipient cartilage damage. Elements of meniscal impingement syndrome are knee hyperextension of at least $5^{\circ}$, articular cartilage injury of the medial femoral condyle of at least Outerbridge grade III, and impingement by the leading edge of the anterior horn of the medial meniscus. The cartilage injuries that we noted in their patient cohort were associated with non-acute stages of this injury and were a result of abnormal contact between the femoral condyle and the meniscal rim. We would suggest that the findings in Takahashi's patients, some of whom were in the reported cohort, as well as some omitted by exclusion criteria for diffuse synovial hypertrophy or chondral fragments, may correlate with findings by McGuire et al. Some of those excluded patients may have had a chondral injury shaped like a horizontal band, as shown in their Fig. 1B, that was located at the junction of the femoral condyle and the meniscal rim and that presented with clinical hyperextension. Unless the synovium is moved away from this area during inspection, and the knee is cycled to hyperextension to correlate the chondral damage with meniscal impingement, this causal relation may easily be overlooked.

In our opinion, the report by Ikeuchi et al. [1] represents meniscal impingement by hyperextension in acute stages of the syndrome, particularly in light of their Fig. 1B image. We also believe that some of their excluded patients may have similar chronic impingement-related chondral damage and diffuse synovial hypertrophy, which are commonly associated with that injury mechanism. 


\section{References}

1. Ikeuchi M, Takahashi T, Tani T. Localized synovial hypertrophy in the anteromedial compartment of the osteoarthritic knee. Arthroscopy. 2005;21:1457-61.
2. McGuire DA, Barber FA, Hendricks SD. Meniscal impingement syndrome. Arthroscopy. 1996;12:675-9.

3. Chow JC, Hantes M, Houle JB. Hypertrophy of the synovium in the anteromedial aspect of the knee joint following trauma: an unusual cause of knee pain. Arthroscopy. 2002;18:735-40.

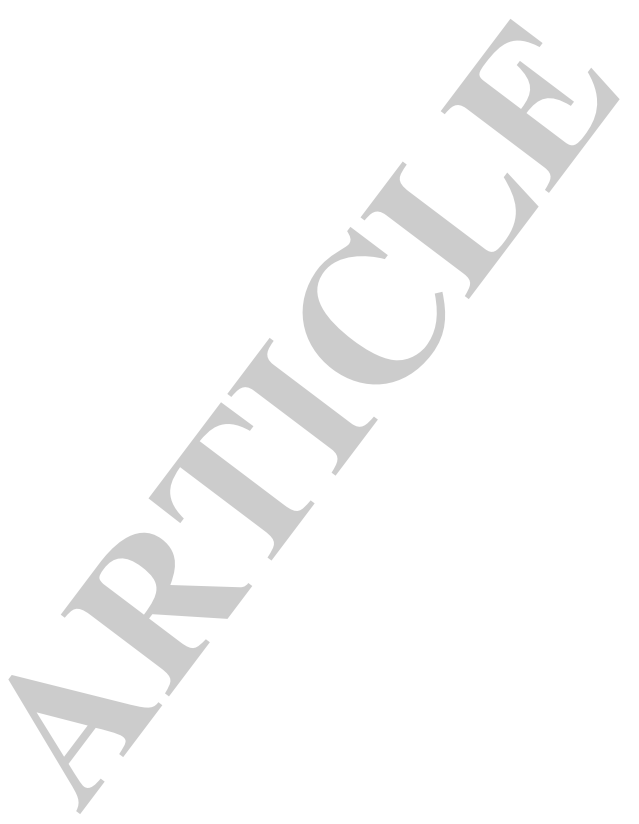

